\title{
Micro-macro FE modelling of damage evolution in laminated composite plates subjected to low velocity impact
}

\author{
N. Li ${ }^{\text {a }}$, P.H. Chen ${ }^{\text {a,* }}$ \\ ${ }^{a}$ State Key Laboratory of Mechanics and Control of Mechanical Structures, Nanjing \\ University of Aeronautics and Astronautics ,Nanjing 210016, China \\ * Corresponding author.
}

Tel: +8613813988209

E-mail address: phchen@nuaa.edu.cn

\begin{abstract}
A phenomenological-based micro-macro mechanical method, comprising the coupling of a 3D continuum damage mechanics (CDM) model with an original micromechanical FE model, is presented to predict low velocity impact response of composite laminates. The key point of the proposed method is the definition and computation of saturation crack density, which is used to scale impact-induced delamination instead of via expensive cohesive zone model based FEM. In order to gain the specific value of the saturation crack density, a micromechanical FE model is established based on the RVE technique. Additionally, the initiation of intralaminar failure including fiber fracture (FF) and inter-fiber fracture (IFF) is evaluated by Puck criteria, and its evolution, with considering effects of the fracture plane angle, is assumed to be controlled by equivalent strains on the fracture plane rather than the classic material principal axis plane. A good agreement between the experimental and simulated results shows correctness and effectiveness of the developed micro-macro
\end{abstract}


mechanical method for predicting impact response and damage using a relatively coarse mesh. Furthermore, significant reduction of computational cost, compared with existing techniques, can be achieved with the novel mesh-independent method.

Keywords: Impact behaviour; Delamination; Saturation crack density; Laminate; Mesh dependency

\section{Introduction}

Impact performance is a particularly crucial consideration in the damage tolerance design of laminated composite structures, whose widespread application are restrained by their high susceptibility to impact loads. The impact-induced damage, typically composed of intralaminar and interlaminar damage, can lead to drastic reductions of structural load-carrying capability despite the fact that it might be barely detected from the surface by visual inspection [1,2]. Readily available methods therefore are strongly required to assess the severity of impact damage in the field of aeronautical engineering.

Recently, continuum damage mechanics (CDM), as a predictive composite method, has been successfully employed to describe the complex failure mechanisms in composite laminates [3]. Firstly, in order to characterize internal damage, a constitutive relation between damaged and intact materials is established by taking into account internal thermodynamically irreversible damage variables associated with possible failure modes. Then damage initiation is predicted with either stress or strain failure criteria. In most of impact experiments, several inner matrix cracks and corresponding inter-fibre fracture (IFF) planes can be obviously observed through the thicknesses direction, which infers that the propagation of matrix cracks located in the inner plies is 
a fundamental cause of generating delamination along both upper and bottom interfaces of the cracked plies $[4,5]$. Thus, the understanding and modeling of matrix cracks and consequent fracture plane should be helpful in establishing a physically based phenomenological model. Puck's fracture criteria [6], which are interactive stress-based criteria valid for unidirectional fibre reinforces polymer composites and take the influence of fracture plane angles into consideration, have been successfully proven their predictive capability in estimating the initiation of intralaminar damage in low velocity impact events $[7,8]$. Even though the fracture plane angles, which should be determined by the true state of stress on the fracture plane, are simplified to a constant value in the literature according to the observation of uniaxial compressive experiments. Once damage initiation, a stiffness degradation law must be defined to represent the damage propagation. The CDM approach degrades material properties until enough energy is absorbed for complete failure as damage is accumulated. With consideration for the strain energy release, a bilinear constitutive relation is widely used to define the evolution of a damage variable representing the failure process in impact events [9-12]. By introducing the characteristic length in FE models, the constant energy release rate per unit area of the crack, to some degree, is generated independently of the mesh size.

The delamination that develops at the interface between two consecutive plies, is one of most important failure modes induced by a low velocity impact, because it is generally considered as a critical factor to the loss of stability of the sublaminate and subsequent stress redistribution that lowers residual strength. For delamination modeling issues, the cohesive zone model (CZM) is widely used and proved its 
effectiveness [10, 13]. However, a general lack of detailed validation of the CZM approach for impact analysis, or discussions of the proper definition of the cohesive layer stiffness and especially the effects of varying the mesh-size on the predicted impact results, is evident. The requirement of extremely refined meshes within the cohesive zone, in order to obtain accurate results, will reduce the stable time increment required and directly result in long calculation time. Moreover, cohesive elements should be placed accurately at each interface between plies, which will also increase the computational amount. Taken together, the mesh sensitivity and simulated interface amounts, as two large obstacles of the impact analyses, must be emphasized and overcome in the current CZM approach.

As mentioned previously, the main objective of this paper is to propose a phenomenological-based, mesh independent micro-macro mechanical method for 3D progressive failure analysis of composite laminates subjected to low velocity impact. Motivating by typical impact damage mechanisms, a crack density parameter, which can be calculated by micromechanical FE models, is employed to simulate delamination with a smeared crack formulation. Finite element analyses have been conducted and the results are compared to the low velocity impact experiments to validate the micro-macro mechanical FE model presented in this paper.

\section{Continuum damage constitutive model}

\subsection{Stress analysis}

According to Mohr's fracture hypothesis, a fracture plane which is parallel to the fiber direction of a unidirectional lamina and defined by the fracture plane angle $\theta$ 
$\left(-90^{\circ} \leq \theta \leq 90^{\circ}\right)$, would be caused by intrinsically brittle failure of composite materials, as illustrated in Fig. 1. Note that coordinates $1-2-3$ and $l-n-t$ are the global lamina coordinate (material principal coordinate) and the fracture plane coordinate, respectively. For orthogonal anisotropy materials, the generalized constitutive equation relating stresses to strains under the coordinate $1-2-3$ and $l-n-t$ can be respectively written in contracted notation as

$$
\boldsymbol{\varepsilon}=\boldsymbol{S}_{0} \boldsymbol{\sigma}, \boldsymbol{\varepsilon}^{\mathrm{fp}}=\boldsymbol{S}_{0}^{\mathrm{fp}} \boldsymbol{\sigma}^{\mathrm{fp}}
$$

Where $S_{0}$ and $S_{0}^{\mathrm{fp}}$ are the initial compliance matrixes in coordinates $1-2-3$ and $l-n-t$, respectively. $\boldsymbol{\sigma}=\left[\sigma_{1}, \sigma_{2}, \sigma_{3}, \tau_{23}, \tau_{13}, \tau_{12}\right]^{\mathrm{T}}, \boldsymbol{\varepsilon}=\left[\varepsilon_{1}, \varepsilon_{2}, \varepsilon_{3}, \gamma_{23}, \gamma_{13}, \gamma_{12}\right]^{\mathrm{T}}$, $\sigma^{\mathrm{fp}}=\left[\sigma_{l}, \sigma_{n}, \sigma_{t}, \tau_{n t}, \tau_{l t}, \tau_{n l}\right]^{\mathrm{T}}$ and $\varepsilon^{\mathrm{fp}}=\left[\varepsilon_{l}, \varepsilon_{n}, \varepsilon_{t}, \gamma_{n t}, \gamma_{l t}, \gamma_{n l}\right]^{\mathrm{T}}$ are the stress and strain components in coordinates $1-2-3$ and $l-n-t$, respectively.

The stresses and strains under a fracture plane coordinate, referred to as the coordinate $l-n-t$, can be transformed from those in the coordinate $l-n-t$ rotating along the fiber direction.

$\boldsymbol{\sigma}^{\mathrm{fp}}=\boldsymbol{T}^{-1} \boldsymbol{\sigma}, \boldsymbol{\varepsilon}^{\mathrm{fp}}=\boldsymbol{T}^{\mathrm{T}} \boldsymbol{\varepsilon}$

Where $\boldsymbol{T}$ is the standard coordinate transformation matrix. Substituting Eq. (2) into Eq. (1) results in

$$
\boldsymbol{S}_{0}^{\mathrm{fp}}=\boldsymbol{T}^{\mathrm{T}} \boldsymbol{S}_{0} \boldsymbol{T}
$$

\subsection{Damage variable matrix}

Distributed microscopic damage in the CDM approach is commonly quantified by the use of an appropriate tensor field that describes the orientation and density of microcracks. A damage variable matrix $\boldsymbol{M}(\boldsymbol{D})$, defined by damage variables $d_{i}$ associated with different internal damage modes, is able to characterize the severity of 
damage and effective fractional reduction of load carrying area. It seems more reasonable, contrary to on the material principal plane, that the impact of the degradation process on the respective stiffness should act on the IFF fracture plane where dramatic reduction in load carrying capacity is directly caused. So the effective stresses is defined in the fracture plane coordinate $l-n-t$ instead of the coordinate $1-2-3$.

$\overline{\boldsymbol{\sigma}}^{\mathrm{fp}}=\boldsymbol{M}^{\mathrm{fp}}(\boldsymbol{D}) \boldsymbol{\sigma}^{\mathrm{fp}}$

Where $\boldsymbol{M}^{\mathrm{fp}}(\boldsymbol{D})=\operatorname{diag}\left[1 / \omega_{l}, 1 / \omega_{n}, 1 / \omega_{t}, 1 / \omega_{n t}, 1 / \omega_{l t}, 1 / \omega_{n l}\right], \omega_{l}=1-d_{l}, \omega_{n}=1-d_{n}$, $\omega_{t}=1-d_{t}, \quad \omega_{n t}=1-d_{n t}, \omega_{l t}=1-d_{l t}, \omega_{n l}=1-d_{n l}$.

The equivalence strain energy is following

$W_{\mathrm{d}}=\frac{1}{2} \overline{\boldsymbol{\sigma}}^{\mathrm{fp}} \boldsymbol{S}_{0}^{\mathrm{fp}} \overline{\boldsymbol{\sigma}}^{\mathrm{fp}}=\frac{1}{2} \boldsymbol{\sigma}^{\mathrm{fp}} \boldsymbol{M}^{\mathrm{fp}}(\boldsymbol{D})^{\mathrm{T}} \boldsymbol{S}_{0}^{\mathrm{fp}} \boldsymbol{M}^{\mathrm{fp}}(\boldsymbol{D}) \boldsymbol{\sigma}^{\mathrm{fp}}=\frac{1}{2} \boldsymbol{\sigma}^{\mathrm{fp}} \boldsymbol{S}_{\mathrm{d}}^{\mathrm{fp}} \boldsymbol{\sigma}^{\mathrm{fp}}$

Therefore, the damaged compliance matrix $S_{\mathrm{d}}^{\mathrm{fp}}$ in the coordinate $l-n-t$ is

$$
\boldsymbol{S}_{\mathrm{d}}^{\mathrm{fp}}=\boldsymbol{M}^{\mathrm{fp}}(\boldsymbol{D})^{\mathrm{T}} \boldsymbol{S}_{0}^{\mathrm{fp}} \boldsymbol{M}^{\mathrm{fp}}(\boldsymbol{D})
$$

Substituting Eq. (3) into Eq. (6), $\boldsymbol{S}_{\mathrm{d}}$ in the coordinate 1-2-3 can be expressed as

$$
\boldsymbol{S}_{\mathrm{d}}=\boldsymbol{T}^{-\mathrm{T}} \boldsymbol{M}^{\mathrm{fp}}(\boldsymbol{D})^{\mathrm{T}} \boldsymbol{T}^{\mathrm{T}} \boldsymbol{S}_{0} \boldsymbol{T} \boldsymbol{M}^{\mathrm{fp}}(\boldsymbol{D}) \boldsymbol{T}^{-1}=\boldsymbol{M}(\boldsymbol{D})^{\mathrm{T}} \boldsymbol{S}_{0} \boldsymbol{M}(\boldsymbol{D})
$$

Where $\boldsymbol{M}(\boldsymbol{D})=\boldsymbol{T} \boldsymbol{M}^{\mathrm{fp}}(\boldsymbol{D}) \boldsymbol{T}^{-1}$ is the damage variable matrix in the coordinate 1-2-3.

\subsection{Failure criteria for intralaminar damage}

Even though Hashin failure criteria have been used extensively in industry, they are not able to accurately predict the matrix damage initiation in compression, due to a lack of considering the influence of transverse compression on matrix shear strength [26]. Therefore, in the present study, the damage model developed by Puck is implemented to model IFF failure. And, it seems reasonable to use the maximum stress criteria, without taking fibre-kinking failure into consideration, to estimate the FF failure, because the fibre compressive failure only occurs in the small-scale area under the impactor in the low velocity impact events. 
The Puck's criteria can calculate the angle-dependent stress exposure factor, $f_{\text {IFF }}(\theta)$, and stress action planes which are all potential fracture planes with an arbitrary angle, $\theta$, against the thickness direction. By this means the action plane with the highest risk of fracture, which is the plane with the highest stress exposure factor, can be identified. According to the potential fracture plane's normal stress, two central expressions of IFF criteria are as follows.

Inter-fiber tension fracture (IFFT):

$\sigma_{n} \geq 0 \rightarrow f_{\mathrm{IFF}}(\theta)=\left[\left(\frac{1}{R_{\perp}^{\mathrm{t}}}-\frac{p_{\perp \psi}^{\mathrm{t}}}{R_{\perp \psi}^{\mathrm{A}}}\right)^{2} \sigma_{n}^{2}(\theta)+\left(\frac{\tau_{n t}(\theta)}{R_{\perp \perp}^{\mathrm{A}}}\right)^{2}+\left(\frac{\tau_{n l}(\theta)}{R_{\perp \|}}\right)^{2}\right]^{\frac{1}{2}}+\frac{p_{\perp \psi}^{\mathrm{t}}}{R_{\perp \psi}^{\mathrm{A}}} \sigma_{n}(\theta)$

Inter-fiber compression fracture (IFFC):

$\sigma_{n}(\theta)<0 \rightarrow f_{\mathrm{IFF}}(\theta)=\left[\left(\frac{p_{\perp \psi}^{\mathrm{c}}}{R_{\perp \psi}^{\mathrm{A}}} \sigma_{n}(\theta)\right)^{2}+\left(\frac{\tau_{n t}(\theta)}{R_{\perp \perp}^{\mathrm{A}}}\right)^{2}+\left(\frac{\tau_{n l}(\theta)}{R_{\perp \|}}\right)^{2}\right]^{\frac{1}{2}}+\frac{p_{\perp \psi}^{\mathrm{c}}}{R_{\perp \psi}^{\mathrm{A}}} \sigma_{n}(\theta)$

With $R_{\perp \perp}^{\mathrm{A}}=\frac{R_{\perp}^{\mathrm{c}}}{2\left(1+p_{\perp \perp}^{\mathrm{c}}\right)}, \frac{p_{\perp \psi}^{\mathrm{t}, \mathrm{c}}}{R_{\perp \psi}^{\mathrm{A}}}=\frac{p_{\perp}^{\mathrm{t}, \mathrm{c}}}{R_{\perp \perp}^{\mathrm{A}}} \cos ^{2} \psi+\frac{p_{\perp \|}^{\mathrm{t}, \mathrm{c}}}{R_{\perp \|}} \sin ^{2} \psi \quad$ and $\cos ^{2} \psi=\frac{\tau_{n t}^{2}}{\tau_{n t}^{2}+\tau_{n l}^{2}}$

Where $R_{\perp}^{\mathrm{t}}$ and $R_{\perp}^{\mathrm{c}}$ are the tensile and compressive strength against the transverse direction. $R_{\perp \|}$ denotes the longitudinal or transverse shear strength. $p_{\perp \|}^{\mathrm{t}}, \quad p_{\perp \perp}^{\mathrm{t}}, \quad p_{\perp \|}^{\mathrm{c}}$ and $p_{\perp \perp}^{\mathrm{c}}$ are the inclination parameters of contour lines of the fracture body. Typically, the present analysis follows the recommendation given in [14] for the specific values of $0.35,0.30,0.27,0.27$ respectively.

The actual fracture plane, following Puck's hypothesis, is the plane with the highest risk of fracture. Consequently, in the general case of three-axial stress states, the calculation of the actual fracture plane angle, $\theta_{\mathrm{fp}}$, turns into a search for the global maximum of the function, $f_{\mathrm{IFF}}(\theta)$, within the interval $\left[-90^{\circ}, 90^{\circ}\right]$. The Golden Section 
Search, as an efficient numerical algorithm for evaluating the global maximum, is adopted to the problem of finding the actual fracture plane angle. When the global maximum value of the function $f_{\mathrm{IFF}}(\theta)$ reaches 1.0 , IFF failure is considered to occur. The obtained fracture angle at this phase is regarded as the actual fracture plane angle, $\theta_{\mathrm{fp}}$, at the material point.

The FF criteria, ignoring the influence of a transverse Poisson's effect, is Fiber tension fracture (FFT):

$$
\sigma_{1} \geq 0 \rightarrow f_{\mathrm{FF}}=\frac{\sigma_{1}}{R_{\|}^{\mathrm{t}}}
$$

Fiber compression fracture (FFC):

$$
\sigma_{1}<0 \rightarrow f_{\mathrm{FF}}=-\frac{\sigma_{1}}{R_{\|}^{\mathrm{c}}}
$$

Where $R_{\|}^{t}$ and $R_{\|}^{c}$ denote the tensile and compressive strengths of the UD laminate in the fiber direction. The FF occurs as soon as the value of $f_{\mathrm{FF}}$ reaches 1.0.

\subsection{Modelling damage propagation}

Once damage initiation, degradation of material stiffness coefficients will be caused by further loading macroscopically, accompanied with release of the strain energy. A evolution law, representing damage propagation in the post-damage initiation phase, is based on the fracture energy dissipated.

\subsubsection{Mesh dependency}

A strong mesh dependency of the FE results in that the energy dissipated decreases upon mesh refinement will be caused by strain localization. Due to the fact that the energy dissipated in the damage propagation phase is proportional to the element size, a 
crack band model is used to reduce the sensitivity to the mesh size [15].

Linear stress-strain behaviour, prior to damage initiation, is assumed. Once a

failure criterion has been satisfied, gradual degradation of material properties follows a linear strain-softening law with a negative slope. The equivalent strain at final failure, $\varepsilon_{\text {eq }}^{\mathrm{f}}$, can be derived from

$\varepsilon_{\text {eq }}^{\mathrm{f}}=\frac{2 G_{\mathrm{c}}}{\sigma_{\mathrm{eq}}^{0} L^{\mathrm{c}}}=\frac{2 g_{\mathrm{c}}}{\sigma_{\mathrm{eq}}^{0}}$

Where $\sigma_{\mathrm{eq}}^{0}$ is the equivalent stress at damage initiation. $G_{\mathrm{c}}$ is the critical fracture energy. $L^{\mathrm{c}}$ is the characteristic length that will keep a constant energy release rate per unit area of the crack. $g_{c}$ is the critical fracture energy density smeared in the characteristic length.

\subsubsection{Internal damage variable}

The bi-linear constitutive relationship results in the following evolution law.

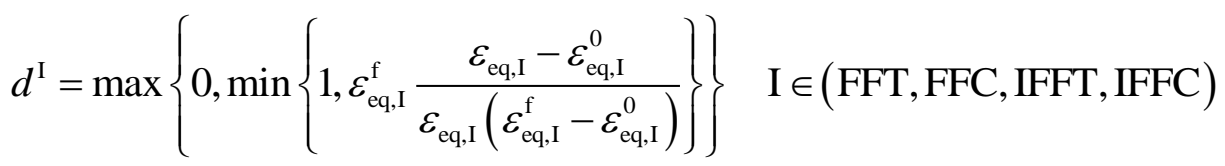

Where $d^{\mathrm{I}}$ represents the internal damage variable corresponding to four damage modes at each time step. $\varepsilon_{\text {eq,I }}, \quad \varepsilon_{\text {eq,I }}^{0}$ and $\varepsilon_{\text {eq,I }}^{\mathrm{f}}$ are the equivalent strains at the current phase, damage initiation and final failure, respectively. Besides, damage irreversibility need to be accounted for with the functional form 'max'.

Obviously, the effect of IFF on material behaviour depends on whether or not crack faces are in contact. If normal stresses, perpendicular to the fracture plane, are tensile $\left(\sigma_{n} \geq 0\right)$, cracks are opened, so no forces can be transmitted as the consequence of no contact between crack faces. Otherwise, cracks are closed and normal forces can be 
transmitted across the cracks. The damage variables associated with IFF and FF failure is given by $d_{n}=\max \left(\frac{\left\langle\sigma_{n}\right\rangle}{\left|\sigma_{n}\right|} d_{n}^{\mathrm{IFFC}}, d_{n}^{\mathrm{IFFT}}\right), d_{l}=d_{l}^{\mathrm{FFT}}+d_{l}^{\mathrm{FFC}}-d_{l}^{\mathrm{FFT}} d_{l}^{\mathrm{FFC}}$

Where $\langle\bullet\rangle$ is the McCauley operator, defined as $\langle x\rangle=(x+|x|) / 2$ for $x \in \mathfrak{R}$.

The damage variable, $d_{t}$, represents damage respect to the $t$-direction that is always perpendicular to the damage axes of IFF and FF failure ( $n$ and $l$ axes). Hence neither IFF nor FF failure would occur along the $t$ damage axis $\left(d_{t}=0\right)$.

The damage variables that affect the shear moduli $d_{i j}(i, j=l, n, t)$ are defined as $d_{n t}=1-\left(1-d_{m}\right)\left(1-d_{t}\right), d_{n l}=1-\left(1-d_{m}\right)\left(1-d_{l}\right)$ and $d_{l t}=1-\left(1-d_{l}\right)\left(1-d_{t}\right)$

With $d_{m}=d_{n}^{\mathrm{IFFT}}+d_{n}^{\mathrm{IFFC}}-d_{n}^{\mathrm{IFFT}} d_{n}^{\mathrm{IFFC}}$

\subsubsection{Equivalent stress and strain}

The interaction of stresses acting on the fracture plane $\left(\sigma_{n}, \tau_{n l}\right.$ and $\left.\tau_{n t}\right)$, based on IFF criteria, is the dominant factor driving the brittle fracture of matrix. For IFF failure modelling, the equivalent stress and corresponding strain are

$$
\sigma_{\text {eq,IFF }}=\sqrt{\left\langle\sigma_{n}\right\rangle^{2}+\tau_{n l}^{2}+\tau_{n t}^{2}}, \quad \varepsilon_{\text {eq, IFF }}=\sqrt{\left\langle\varepsilon_{n}\right\rangle^{2}+\gamma_{n l}^{2}+\gamma_{n t}^{2}}
$$

Similarly, the equivalent stress and strain for FF failure is given by

$$
\sigma_{\mathrm{eq}, \mathrm{FF}}=\sqrt{\sigma_{1}^{2}}, \quad \varepsilon_{\mathrm{eq}, \mathrm{FF}}=\sqrt{\varepsilon_{1}^{2}}
$$

\subsubsection{Delamination modelling by intralaminar matrix cracks}

Experimental evidence indicates that under a given impact energy, intralaminar matrix cracks, as the initial damage mode, propagate into the nearby interface and then coalesce into one interface crack, namely delamination [16-18]. Thus, an interlaminar damage model is proposed by means of an original damage mechanics (DM) approach. 
If the angle of IFF fracture plane at the material point is equal to $90^{\circ}$, delamination occurs. Otherwise, no delamination would be observed until the density of intralaminar matrix cracks approaches a saturation damage state. Thus, a saturation crack density parameter, $\rho_{\text {crack }}$, is employed to quantify the critical number of intralaminar matrix cracks required to generate the interface crack per unit length $[19,20]$.

Generally, the semi-empirical approach [21] for predicting the specific value of above saturation crack density parameter depends heavily on the results of impact experiments, and the influence of stacking sequences and impact energies on this parameter is lack of detailed validation. An original micromechanical FE method, therefore, is proposed to determine the value of saturation crack density in Section 3.1.

In this paper, the presented model is modified to allow multiple matrix cracks, one fibre fracture surface and one interface crack per element. Considering the fracture energy dissipated by multiple cracks, a quadratic interaction function for the fracture energy in the mix-mode case is established.

$\left(\frac{g_{n} L_{\mathrm{IFF}}^{\mathrm{c}}}{n_{\text {crack }} G_{2 \mathrm{c}}^{\mathrm{k}}}\right)^{2}+\left(\frac{g_{n l} L_{\mathrm{IFF}}^{\mathrm{c}}}{n_{\text {crack }} G_{12 \mathrm{c}}}\right)^{2}+\left(\frac{g_{n t} L_{\mathrm{IFF}}^{\mathrm{c}}}{n_{\text {crack }} G_{23 \mathrm{c}}}\right)^{2}=1$

Where the superscript $\mathrm{k}(\mathrm{k}=\mathrm{t}, \mathrm{c})$ denotes the tensile or compressive load. $L_{\mathrm{IFF}}^{\mathrm{c}}$ is the characteristic element lengths for IFF, and is defined as

$$
L_{\mathrm{IFF}}^{\mathrm{c}}=\left\{\begin{array}{cc}
l_{z} & \text { for }\left|\theta_{\mathrm{fp}}\right|=90^{\circ} \\
l_{y} \cos \left(\theta_{\mathrm{fp}}\right) & \text { for }\left|\theta_{\mathrm{fp}}\right| \neq 90^{\circ}
\end{array}\right.
$$

Where $l_{y}$ and $l_{z}$ corresponds to the FE length measured in the global coordinate system along $y$ - and $z$-direction, respectively. In addition, $n_{\text {crack }}$ quantifies the number of matrix cracks at saturation per element, which is calculated by 
$n_{\text {crack }}=\left\{\begin{array}{cc}1 & \text { for }\left|\theta_{\mathrm{fp}}\right|=90^{\circ} \\ \rho_{\text {crack }} l_{y} & \text { for }\left|\theta_{\mathrm{fp}}\right| \neq 90^{\circ}\end{array}\right.$

In Eq. (18), it illustrates that once the fracture energy of $n_{\text {crack }}$ intralaminar microcracks is completely dissipated, delamination might be generated by the propagation of those intralaminar cracks with respect to the through-thickness direction. Hence delamination modelling can be achieved, in one element, by scaling fracture energy release rates of the number of intralaminar cracks at the saturation damage state.

An interaction between intralaminar and interlaminar damage is able to be considered in the proposed model as followings. Conventionally, the first type of damage in impacted composite laminates is matrix cracking. The initiation of matrix cracking can be captured by IFF criteria as soon as the global maximum value of the function $f_{\mathrm{IFF}}(\theta)$ reaches 1.0. Afterwards, for the elements with matrix failure, the reduction in their load carrying capacity is simulated by a damage variable matrix $\boldsymbol{M}(\boldsymbol{D})$, which is defined by damage variables $d_{i}$ associated with different internal damage modes. Further increased applied loads will be redistributed from the degrading elements to the neighbouring ones, and meanwhile result in the increase of the equivalent strain. This load redistribution may cause the initiation of the intralaminar damage, estimated by failure criteria, in neighbouring elements, which can be seen as the macroscopic propagation of the intralaminar damage in a material. According to Eq. (13), the increase of the equivalent strain will clearly give rise to the increase of the damage variables $d_{i}$ corresponding to IFF failure. Because the presented model is modified to simulate multiple matrix cracks (quantified by $n_{\text {crack }}$ ) at saturation per element (Eq. (18)), above increase of the damage variables indicates the generation of 
more matrix cracks, accompanied with releasing more energy. Once the fracture energy of $n_{\text {crack }}$ matrix cracks is completely dissipated $\left(d_{i}=1\right)$, it denotes the complete generation of saturation matrix cracks. Thus, the interlaminar damage (i.e. delamination) would quickly occur based on the failure mechanism indicating that the interlaminar cracks develop and coalesce into one interface crack. The shear moduli $G_{13}$ and $G_{23}$, corresponding to delamination, are completely degraded. Then, more loads will be transferred from the elements with delamination to the neighbouring ones, which may more easily cause the initiation of the intralaminar damage and accelerate the occurrence of the interlaminar damage in the surrounding area.

\subsubsection{Final failure strain}

The strain energy density released in the characteristic length, due to complete IFF failure, $g_{i}^{\mathrm{f}}$ can be given in

$$
g_{i}^{\mathrm{f}}=\int_{0}^{\varepsilon_{i}^{\mathrm{f}}} \sigma_{i} \mathrm{~d} \varepsilon \approx \frac{1}{2} \sigma_{i}^{0} \varepsilon_{i}^{\mathrm{f}}=\frac{1}{2} \sigma_{i}^{0} \beta_{i} \varepsilon_{\mathrm{eq}}^{\mathrm{f}} \quad(i=n, n l, n t)
$$

Where $\sigma_{i}^{0}$ is the stress component acting on the fracture plane at IFF failure initiation. $\beta_{i}$ denotes the mix-mode ratio and can be expressed as

$$
\beta_{n}=\frac{\left\langle\varepsilon_{n}\right\rangle}{\varepsilon_{\text {eq }}}, \quad \beta_{n l}=\frac{\gamma_{n l}}{\varepsilon_{\text {eq }}}, \quad \beta_{n t}=\frac{\gamma_{n t}}{\varepsilon_{\text {eq }}}
$$

Hence, the equivalent strain at final IFF failure is

$$
\varepsilon_{\text {eq,IFF }}^{\mathrm{f}}=\left\{\begin{array}{ll}
\frac{2}{l_{z}} \Delta & \text { for }\left|\theta_{\mathrm{fp}}\right|=90^{\circ} \\
\frac{2 \rho_{\text {crack }}}{\cos \left(\theta_{\mathrm{fp}}\right)} \Delta & \text { for }\left|\theta_{\mathrm{fp}}\right| \neq 90^{\circ}
\end{array} \quad \Delta=\left[\left(\frac{\sigma_{n}^{0} \beta_{n}}{G_{2 \mathrm{c}}^{\mathrm{k}}}\right)^{\zeta}+\left(\frac{\tau_{n l}^{0} \beta_{n l}}{G_{12 \mathrm{c}}}\right)^{\zeta}+\left(\frac{\tau_{n t}^{0} \beta_{n t}}{G_{23 \mathrm{c}}}\right)^{\zeta}\right]^{-1 / \zeta}\right.
$$

Due to instantaneous energy release once the occurrence of FF failure, the equivalent strain $\varepsilon_{\text {eq,FF }}^{\mathrm{f}}$, corresponding to the final failure strain for fibre damage, is 
quite close to the strain at failure onset, $\varepsilon_{\mathrm{eq}, \mathrm{FF}}^{0}$. It seems reasonable to assume that the material is completely destroyed once the FF failure is detected $\left(\varepsilon_{\mathrm{eq}, \mathrm{FF}}^{\mathrm{f}} \approx \varepsilon_{\mathrm{eq}, \mathrm{FF}}^{0}\right)$.

\section{Numerical analysis of impact event and experimental verification}

\subsection{Saturation crack density computation}

The increase of the transverse crack density is actually a crack initiation process, which continues until the crack density reaches a saturation state. After the initiation of a transverse crack, the load instantaneously transfers from this crack to neighboring plies, which causes the decrease of stress field surrounding the free surface of the open crack. However, the stress will increase again with increasing distance from the crack surface. Consequently, the second crack will be generated once the stress, somewhere away from the crack, satisfies the stress level for matrix microcracking. In other words, the saturation crack distance might be represented by the minimum distance between the existing crack and the position where the transverse stress achieves steady state. The position of the second crack at saturation state is theoretically equal to the position where the stress ratio changes to 1.0 .

A micromechanical finite element model based on the RVE technique is set up to compute the minimum distance. A 1/4 model with one central through crack, simulated by the release of crack surface nodes, has been established by taking into account the geometric symmetry, as shown in Fig. 2. A tensile displacement $d$ is applied at the end, while symmetry constraints are applied on two symmetrical planes, namely plane A-O-B and A-O-C.

In the RVE model, load needs to be applied perpendicular to the surface of the 
crack which is running along the fibre direction on the 1-2 plane. In other words, the load is applied along the transverse direction of a lamina. Hence, for the convenience of modeling, the angle of the middle cracked ply would be better equal to $90^{\circ}$. In order to study the influence of ply stiffness on the value of the saturation crack density, six configurations varying from the stacking sequence of neighboring plies or thickness of the cracked ply, are designed. Fig. 3 (a) gives the distribution of transverse stress at the cross section A-O-C of the laminates manufactured with HS300/ET223. Note that the stress ratio is calculated through dividing the current stress by that in far field.

By comparing the results of laminates [45/ $\underline{90} / 45],[0 / \underline{90} / 0],\left[45_{3} / 0 / \underline{90} / 0 / 45_{3}\right]$ and [0/90/45], the positions, where stress ratios change to 1.0, are quite close, while those are different after comparing the results of laminates $[0 / \underline{90} / 0],[0 / \underline{902} / 0]$ and $[0 / \underline{90} 4 / 0]$. It indicates that the saturation crack distance is affected significantly by thickness of the cracked ply rather than stiffness of neighboring plies. Hence, there seems little difference on the value of the saturation crack density, if the thickness of the cracked ply is identical (generally was set equal to the ply thickness).

RVE models of laminates [0/90/0] are established to obtain the distribution of transverse stress at cracked plies that are manufactured with test materials (Table 1). Specific values of the saturation crack distance, as shown in Fig. 3 (b), are $0.49 \mathrm{~mm}$ and $0.20 \mathrm{~mm}$ for materials HS300/ET223 and T300/NY9200Z respectively. That is, corresponding values of saturation crack densities are $2.05 \mathrm{~mm}^{-1}$ and $5.00 \mathrm{~mm}^{-1}$.

\subsection{Low velocity impact tests}

To validate the proposed model, the low velocity response of laminated composite plates, with different stacking sequences, materials and geometrical dimensions, is 
investigated at various impact energies by using an instrumented drop tower. Two impact test cases, referred to as tests \#1 and \#2, are chosen. The specimens are manufactured with HS300/ET223 and T300/NY9200Z graphite/epoxy prepreg plies for test \#1 [22] and test \#2, respectively. The material properties of test materials are listed in Table 1 , where the fiber volume fraction is identically $64 \%$. The stacking sequences of composite laminates in tests $\# 1$ and $\# 2$ are $\left[0_{3} / 45 /-45\right]_{S}$ and $[45 / 0 /-45 / 90]_{4 S}$ (quasi-isotropic lay-ups) respectively, defined by taking the $0^{\circ}$ fiber orientation aligned with the longer in-plane dimension.

Rectangular specimens in test \#1, with $65 \mathrm{~mm} \times 87.5 \mathrm{~mm}$ in-plane dimensions and a nominal thickness of $3.2 \mathrm{~mm}$ are simply supported on a steel plate with a $45 \mathrm{~mm} \times$ $67.5 \mathrm{~mm}$ rectangular cut-out. The laminates are impacted with various energies gained by varying the drop height of a hemispherical impactor, with a mass of $5 \mathrm{~kg}$ and a diameter of $12.5 \mathrm{~mm}$. For test \#2, taking the ASTM D7136 test standard as a reference, the specimens with $150 \mathrm{~mm} \times 100 \mathrm{~mm}$ in-plane dimensions and a nominal thickness of about $4 \mathrm{~mm}$ are placed over a flat support fixture base with a $125 \mathrm{~mm} \times 75 \mathrm{~mm}$ rectangular cut-out. The support fixture base has four rubber-tipped clamps which clamp the specimen during impact. The impacts are introduced onto the specimen using a $16 \mathrm{~mm}$ diameter hemispherical impactor made of hardened steel, and each specimen only receives one impact. At a fixed height of $1130 \mathrm{~mm}$, the impact energy is controlled by varying mass.

\subsection{Simulation of impact event}

The Abaqus/Explicit code is used to run the numerical FE model where the CDM model, together with the proposed numerical simulation method of impact-induced 
interlaminar damage, is implemented by a user-defined material subroutine (VUMAT). Appropriate geometrical models are built, and kinematic and loading boundary conditions are defined to represent the experimental set up.

The FE model simulates, in the way shown in Fig.4, the geometry described in section 3.2. The numerical laminates, discretized with one element through-thickness per each ply, are represented by eight-node reduced integration brick elements, C3D8R. And, interface elements are not used. Different mesh size is introduced in different regions, to reduce the computing time, from $1 \mathrm{~mm} \times 1 \mathrm{~mm}$ elements in the central target window up to coarser mesh size elements outside the clamped window. Moreover, the Enhanced Hourglass Control approach [27], one of control methods for suppressing hourglassing, is selected for all simulations. Both the supporting plate and only the lower half of the semi-spherical tup of the impactor are discretized by means of rigid elements.

The region of the supporting plate is simulated by constraining all freedom degrees of the nodes. Particularly, all the corners of the specimen are constrained their $x$-, $y$ - and $z$-direction to consider the influence of four rubber-tipped clamps during impact. The mass and initial velocities, which match the impact test, are assigned to the impactor. For contact between the surface of the metal parts and the composite laminate, a friction coefficient of 0.3 is adopted [23].

The input material properties used in the numerical simulation is partly presented in Table 1. Besides, due to identical fracture modes, the critical fracture energy release rates corresponding to transverse tension and in-plane shear $\left(G_{2 \mathrm{c}}^{\mathrm{t}}\right.$ and $\left.G_{12 \mathrm{c}}\right)$ might be replaced by mode I and mode II fracture energy release rates that can be measured in standard tests. Meanwhile, the critical fracture energy release rates corresponding to interlaminar shear, $G_{23 \mathrm{c}}$, is assumed to be equal to $G_{12 \mathrm{c}}$. The values of critical fracture 
energy release rates and saturation crack densities are listed in Table 2.

\subsection{Experimental verification}

\subsubsection{Prediction of impact structural response}

The impact force histories obtained by test \#1 and those predicted by the FE models are shown in the graphs of Fig. 5 for three impact energies including 2J, 4J and 8J. Good agreement is observed between measured and predicted results, whereas predicted peak loads somewhat higher than those measured experimentally. The maximum forces of $2.5 \mathrm{kN}, 3.3 \mathrm{kN}$ and $4.7 \mathrm{kN}$ predicted numerically, corresponding to impact energies of $2 \mathrm{~J}, 4 \mathrm{~J}$ and $8 \mathrm{~J}$, closely match the measured values of $2.3 \mathrm{kN}, 3.1 \mathrm{kN}$ and $4.6 \mathrm{kN}$. We note that the error of maximal $8.7 \%$ appears in the simulation of the specimen for the $2 \mathrm{~J}$ impact test. In both loading and unloading phases, the impact force-time curves predicted also show a reasonably good match with those observed in test \#1. A sudden drop in force which might be associated to the initiation and further propagation of fibre fracture, for instance, is recorded at a peak load both experimentally and numerically during the $8 \mathrm{~J}$ impact test.

The impact force-displacement curves (see in Fig. 6) are translated from the force-time histories presented in Fig. 5. There is a similar slope for experimental and predicted curves under respective impact energy, until the maximum impact force is reached during the loading phase of the impact events. However, in the unloading phase, the slope of force-displacement curves per energy is in better agreement with increasing impact energy. Accompany with the damage propagation in the laminate, dramatic fluctuation of force-displacement curve appears, due to the gradual stiffness reduction that is caused by its severe local softening behaviour at the failure location. In addition, the impact force during the projectile rebound is lower than that in the loading phase, also as a consequence of the reduction of the laminate stiffness. Generally, all 
impact force-displacement curves exhibit distinctly nonlinear characteristics.

\subsubsection{Prediction of impact damage}

For now, it demonstrates that post-impact damage is essential for the prediction of the compression after impact (CAI) strength of the composite aeronautical structures. Comparisons between delamination images obtained by non-destructive evaluation (NDE) (including X-ray radiographs and C-scan) and FEM at different impact energies are illustrated in Table 3. Note that the dimensions of captured square regions are 65 $\mathrm{mm} \times 87.5 \mathrm{~mm}$ and $60 \mathrm{~mm} \times 60 \mathrm{~mm}$ for tests $\# 1$ and $\# 2$ respectively.

The predicted envelopes of saturation matrix cracks, namely delamination, can correctly reproduce peculiar features of the impact-induced interface failure of the laminates, such as the shape of an ellipse, of which longer axis is along the longitudinal direction, presented by the projected delamination area. Due to the fact that the delamination is simulated approximately at the mid-plane of each ply rather than at the plies' interface, and meanwhile delamination failure that is caused by the relative motion between debonding locations is out of the consideration in the proposed damage model, the accuracy of the model at capturing delamination features is inevitably more and more lower as increasing impact energies. However, comparisons between measured and predicted results for absorbed energy and dimensions of projected delamination area, as illustrated in Fig. 7 and Fig. 8, seem favourable for laminates with various impact energies, geometrical dimensions, clamping conditions and stacking sequences. The predicted points and fitted curves are almost within the data bands formed by measured points, which validates the failure formulation proposed. 
Conventionally, the first type of impact damage is matrix cracking, which triggers subsequent interface and fibre failure during the impact tests. The distribution of IFF fracture plane angles, which is closely related to current stress states, is investigated by taking an example of the laminate $\left[0_{3} / 45 /-45\right]_{S}$ under the impact energy of $4 \mathrm{~J}$, in the graphs of Fig. 9. The value of fracture plane angle in one ply is defined in the global lamina coordinate system 1-2-3. In the area of plies adjacent to the impact surface, such as the 1st ply in Fig. 9 (a), its fracture plane angle is predicted with values larger than $50^{\circ}$, it can be seen that IFFC failure occurs in the plies adjacent to the impact surface due to impactor's pressure, and the extent of IFFC failure is limited in the radius of the impactor. The distribution of shear stresses within a laminated composite approximately presents a parabolic shape under impact loads, which means plies in the middle of a laminate primarily withstand shear stresses (Fig. 9 (b)). Thus, IFF failure owing to shear stresses is the main matrix fracture type in the middle plies through-thickness, which results in fracture plane angles around $\pm 45^{\circ}$. For the plies away from the impactor, their actual fracture plane angles are approximately equal to $0^{\circ}$ which is the angle of the fracture plane perpendicular to the laminate plane, as shown in Fig. 9 (c). This phenomenon is mainly due to the fact that the in-plane transverse tensile stress, introduced by the bending moment from the supporting boundary, is the dominant stress causing the critical matrix cracking during impact. Therefore, the transverse matrix cracks in the impact backside of a laminated composite are belong to tensile matrix fracture. Furthermore, the IFF failure in most plies is caused by shear stresses, while in only a small number of plies adjacent to the impact surface or back surface, the damage 
is caused by transverse stresses. But the extent of tensile matrix failure at the backside is larger than the one in any other ply. The simulated distribution of intralaminar microcracking is consistent with the experimental observation [16-18].

\subsubsection{Validation of the mesh independency}

Impact force-time histories, force-displacement curves, and the damage areas of laminates $\left[0_{3} / 45 /-45\right]_{S}$ in test $\# 1$ are plotted for these three numerical targets with different mesh densities. FE models are discretized with in-plane dimensions of $2 \mathrm{~mm} \times$ $2 \mathrm{~mm}, 1.5 \mathrm{~mm} \times 1.5 \mathrm{~mm}$ and $1 \mathrm{~mm} \times 1 \mathrm{~mm}$ (in both cases $0.32 \mathrm{~mm}$ thick), respectively. The results presented in Fig. 10 and Fig. 11 show that mesh-size independent impact damage analysis can be successfully achieved with the proposed method during $4 \mathrm{~J}$ impact event. Both qualitative (extent and shape of projected delamination areas) and quantitative (force-time histories, force-displacement curves and absorbed energies) results are not sensitive to the mesh-size change. Slight differences, reflected in the envelope of matrix damage and oscillation of predicted curves, between the results obtained for the different mesh densities can be attributed to the deterioration of stress computing at Gauss integral points and interaction response between contacting surfaces with increasingly coarser FE sizes.

\section{Discussions}

A predictive composite method for accurately describing complex interlaminar and intralaminar fracture mechanisms is required in modelling impact in laminated composites. It classically comprises the coupling of a mesoscale in-plane damage model with interface cohesive elements by considering intralaminar and interlaminar damage 
types separately. However, when using interface cohesive elements, the requirement of extremely refined meshes and application in each ply interface will significantly reduce the computational efficiency. The proposed method, featuring an original CDM approach, adopts Puck's phenomenological-based failure criteria to characterize intralaminar fracture failure, and meanwhile obtain the specific fracture plane angle that can imitate delamination. Based on the impact-induced delamination failure mechanism, approximated modelling of delamination failure can be achieved by the formation of multiple saturation matrix cracks instead of using interface cohesive elements. Thus, a parameter, which quantifies this saturation level for intralaminar matrix cracks per element, is introduced to dissipate the correct amount of fracture energy associated with distributed damage in the form of multiple cracks.

A micromechanical method, contrary to an inverse approach depending heavily on experimental results, is developed for predicting the value of this saturation crack density parameter. Based on the assumption that the saturation crack distance might be represented by the position where the transverse stress achieves steady state, only the distribution of transverse stress in the cracked ply needs to be obtained in the proposed method. It will clearly result in a much reduced computational cost, regardless of considering energy release in the initiation and propagation process of a new crack. It should be noted that the saturation matrix cracks will introduce delamination on the upper and lower interface of the cracked ply, due to the identical stress distribution on both interface.

A good agreement between the experimental and simulated results shows 
correctness and effectiveness of the developed micro-macro mechanical method for predicting the structural response and damage status of low velocity impact events. Moreover, results for different mesh-densities indicated that the approach by introducing a characteristic length of the element could alleviate the mesh dependency at the stage of material damage evolution.

It should be emphasized that the delamination is simulated approximately at the mid-plane of each ply rather than the interface between adjacent plies. Also, the delamination caused by stiffness mismatch of adjacent plies, such as edge impact on composite laminates $[24,25]$, and the relative motion between two debonding parts, indeed, might not be well simulated. However, this approximated method of modelling delamination would be suitable for predicting classical out-of-plane impact damage of laminated composites, especially those with much more plies. Furthermore, in contrast to the CZM approach that can accurately predict each delaminated interface, the proposed modelling approach may hardly obtain the actual size of delamination at each interface, as the delamination in this method represents both the upper and lower interface of the ply, and it is simulated approximately at the mid-plane of each ply rather than at the plies' interface like cohesive elements.

However, the results presented in this paper demonstrate its predictive capabilities, both qualitatively and quantitatively. In addition, it could result in decreasing the amount of elements and improving the computational efficiency, due to no requirement of extra interface elements.

\section{Concluding remarks}


In this paper, a phenomenological-based micro-macro mechanical method comprising a three-dimensional anisotropic CDM model, including damage characterization, damage initiation criterion and damage evolution law, and a micromechanical FE model, to compute saturation matrix crack densities, has been proposed and successfully used to analyze low-velocity impact damage of composite laminates. The following conclusions can be drawn from the present study:

Based on the assumption that IFF failure directly causes a dramatic reduction in load carrying capacity on the fracture plane rather than on the plane corresponding to the principal material direction, a damage variable matrix is defined in the fracture plane coordinate system and then deduced under the global lamina coordinate system. The linear progressive damage evolution law controlled by the equivalent strains acting on the fracture plane is established according to the viewpoint of strain energy release.

A micromechanical finite element model based on the RVE technique has been proposed to obtain the saturation matrix crack density, which is considered as a significant parameter to simulate delamination by multiple intralaminar matrix cracks for the purpose of improving computational efficiency. Rather good agreement has been observed between experiments and predictions in terms of impact force-time histories, impact force-displacement curves, absorbed impact energies and typical failure morphology of composite laminates subject to low velocity impact damage.

Results for different mesh-densities indicated that the approach by introducing a characteristic length of the element could alleviate the mesh dependency at the stage of material damage evolution, but it seems impossible to completely solve the problem of 
mesh dependence because of the deterioration of the kinematic representation with increasingly coarser mesh sizes.

The analysis of the distribution of IFF plane angles indicates that most plies, positioned in the middle of a laminate, suffer shear matrix failure owing to shear stresses, which results in the fracture plane angles around $\pm 45^{\circ}$. However, in the area of plies adjacent to the impact surface and back surface, their IFF is mostly caused by transverse stresses. IFFC failure would occur in the plies adjacent to the impact surface due to the impactor's pressure, while IFFT failure, caused by in-plane transverse tensile stress, is observed in plies adjacent to the impact back surface. Hence the actual fracture plane angles for matrix cracks in plies adjacent to the impact surface and back surface are larger than $50^{\circ}$ and approximately equal to $0^{\circ}$, respectively.

\section{Acknowledgments}

This research is supported by the National Science Foundation of China (No. 11572152).

\section{References}

[1] Hull D, Shi YB. Damage Mechanism Characterization in Composite Damage Tolerance Investigations. Composite Structures 1993; 23: 99-120.

[2] Soutis C, Curtis PT. Prediction of the post-impact compressive strength of CFRP laminated composites. Composite Science and Technology 1996; 56: 677-684.

[3] Maimi P, Mayugo J A, Camanho P P. A three-dimensional damage model for transversely isotropic composite laminates. Journal of Composite Materials, 2008, 42: 2717-2745.

[4] Bouvet C, Castanie B, Bizeul M, et al. Low velocity impact modelling in laminate 
composite panels with discrete interface elements. International Journal of Solids and Structures 2009; 46: 2809-2821.

[5] Petit S, Bouvet C, Bergerot A, et al. Impact and compression after impact experimental study of a composite laminate with a cork thermal shield. Composite Science and Technology 2007; 67: 3286-3299.

[6] Puck A, Schurmann H. Failure analysis of FRP laminates by means of physically based phenomenological models. Composite Science and Technology 1998; 58(10): 1045-1067.

[7] Hinton MJ, Kaddour AS, Soden PD. A comparison of the predictive capabilities of current failure theories for composite laminates judged against experimental evidence. Composite Science and Technology 2002; 62: 1725-1797.

[8] Soden PD, Kaddour AS, Hinton MJ. Recommendations for designersand researchers resulting from the world-wide failure exercise. Composite Science and Technology 2004; 64: 589-604.

[9] Faggiani A, Falzon BG. Predicting low-velocity impact damage on a stiffened composite panel. Composites: Part A 2010; 41: 737-749.

[10] Shi Y, Swait T, Soutis C. Modelling damage evolution in composite laminates subjected to low velocity impact. Composite Structures 2012; 94: 2902-2913.

[11]Falzon BG, Apruzzese P. Numerical analysis of interlaminar failure mechanisms in composite structures. Part I: FE implementation. Composite Structures 2011; 93(2): 1039-1046.

[12]Falzon BG, Apruzzese P. Numerical analysis of interlaminar failure mechanisms in composite syructures. Part II: Applications. Composite Structures 2011; 93(2): 1047-1053. 
[13] Aymerich F, Dore F, Priolo P. Prediction of impact-induced delamination in cross-ply composite laminates using cohesive interface elements. Composites Science and Technology; 2008, 68: 2383-2390.

[14]Deuschle HM. 3D failure analysis of UD fibre reinforced composites: Puck's theory within FEA. Germany: University Stuttgart, 2010.

[15]Bazant ZP, Oh BH. Crack band theory for fracture of concrete. Mater Struct 1983; $16: 155-77$.

[16]Mitrevski T, Marshall IH, Thomson R. The influence of impactor shape on the damage to composite laminates. Composite Structures 2006; 76: 116-122.

[17]Choi HY, Downs RJ, Chang FK. A new approach toward understanding damage mechanisms and mechanics of laminated composites due to low-velocity impact, part I. Journal of Composite Materials 1991; 25(8): 992-1011.

[18]Choi HY, Downs RJ, Chang FK. A new approach toward understanding damage mechanisms and mechanics of laminated composites due to low-velocity impact, part II. Journal of Composite Materials 1991; 25(8): 1012-1038.

[19]Nairn JA. Matrix Microcracking in Composites. Polymer Matrix Composites 2000; 2: 403-432.

[20]Talreja R, Singh CV. Damage and Failure of Composite Materials. Cambridge: Cambridge University Press, 2012.

[21]Raimondo L, Iannucci L, Robinson P, et al. A progres-sive failure model for mesh size independent FE analysis of composite laminates subject to low-velocity impact damage. Composite Science and Technology 2012; 72: 624-632. 
[22]Feng D, Aymerich F. Finite element modelling of damage induced by low-velocity impact on composite laminates. Composite Structures 2014; 108: 161-171.

[23] Gonzalez EV, Maimi P, Camanho PP, et al. Simulation of drop weight impact and compression after impact tests on composite laminates. Composite Structures 2012; 94: 3364-3378.

[24]Malhotra A, Guild F, Pavier M. Edge impact to composite laminates: experiments and simulations. J Mater Sci 2008; 43: 6661-6667.

[25] Ostre B, Bouvet C, Lachaud F, et al. Edge impact modeling on stiffened composite structures. Composite Structures 2015;126: 314-328.

[26] Davila CG, Camanho PP, Rose CA. Failure criteria for FRP laminates. Journal of Composite Materials 2005; 39(4): 323-345.

[27] ABAQUS. ABAQUS 6.10 Documentation. Dessault systemes. 2010. 
Table 1 Material properties of HS300/ET223 and T300/NY9200Z laminates

\begin{tabular}{|c|c|c|c|c|c|c|c|c|c|c|c|}
\hline \multirow{2}{*}{ Material } & \multicolumn{4}{|c|}{ Modulus/GPa } & \multicolumn{2}{|c|}{ Poisson's ratio } & \multicolumn{5}{|c|}{ Strength/MPa } \\
\hline & $E_{1}$ & $E_{2}=E_{3}$ & $G_{12}=G_{13}$ & $G_{23}^{a}$ & $v_{12}=v_{13}$ & $v_{23}$ & $R_{\|}^{t}$ & $R_{\|}^{c}$ & $R_{\perp}^{t}$ & $R_{\perp}^{c}$ & $R_{\perp \|}$ \\
\hline HS300/ET223 [22] & 122 & 6.2 & 3.9 & 2.1 & 0.35 & 0.5 & 1850 & 1470 & 29 & 140 & 61 \\
\hline T300/NY9200Z & 137 & 9.3 & 5.3 & 3.6 & 0.31 & 0.3 & 1747 & 1357 & 67 & 170 & 124 \\
\hline
\end{tabular}

${ }^{\mathrm{a}}$ indicates the estimated value, instead of the experiment data of material property. 
Table 2 Critical fracture energy release rates and saturation crack densities

\begin{tabular}{cccc}
\hline Material & $G_{2 \mathrm{c}}^{\mathrm{t}}(\mathrm{N} / \mathrm{mm})$ & $G_{12 \mathrm{c}}=G_{23 \mathrm{c}}(\mathrm{N} / \mathrm{mm})$ & $\rho_{\text {crack }}\left(\mathrm{mm}^{-1}\right)$ \\
\hline HS300/ET223 & 0.52 & 0.92 & 2.05 \\
T300/NY9200Z & 0.175 & 0.348 & 5.00 \\
\hline
\end{tabular}


Table 3 Comparisons between delamination images obtained by NDE and FEM

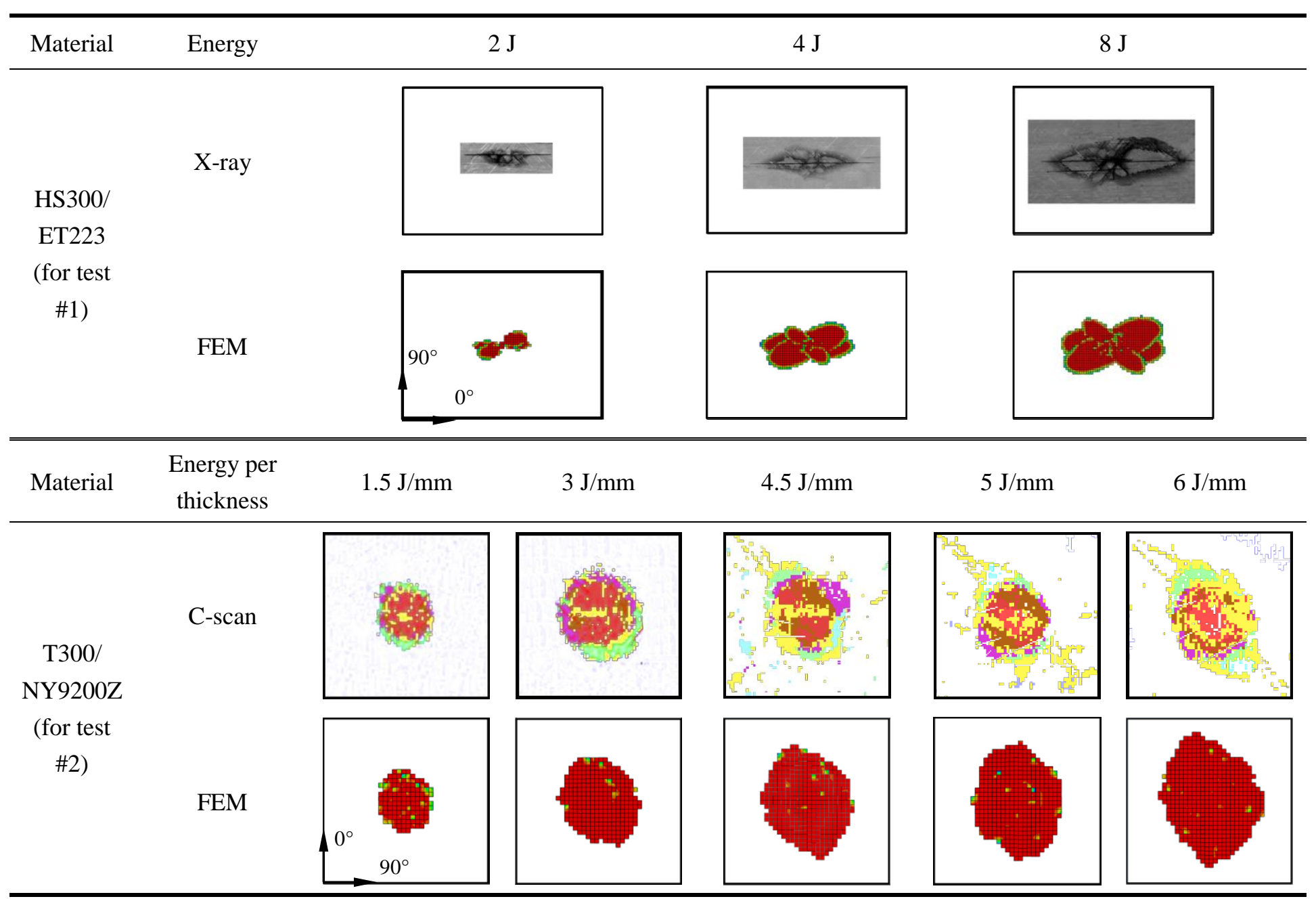




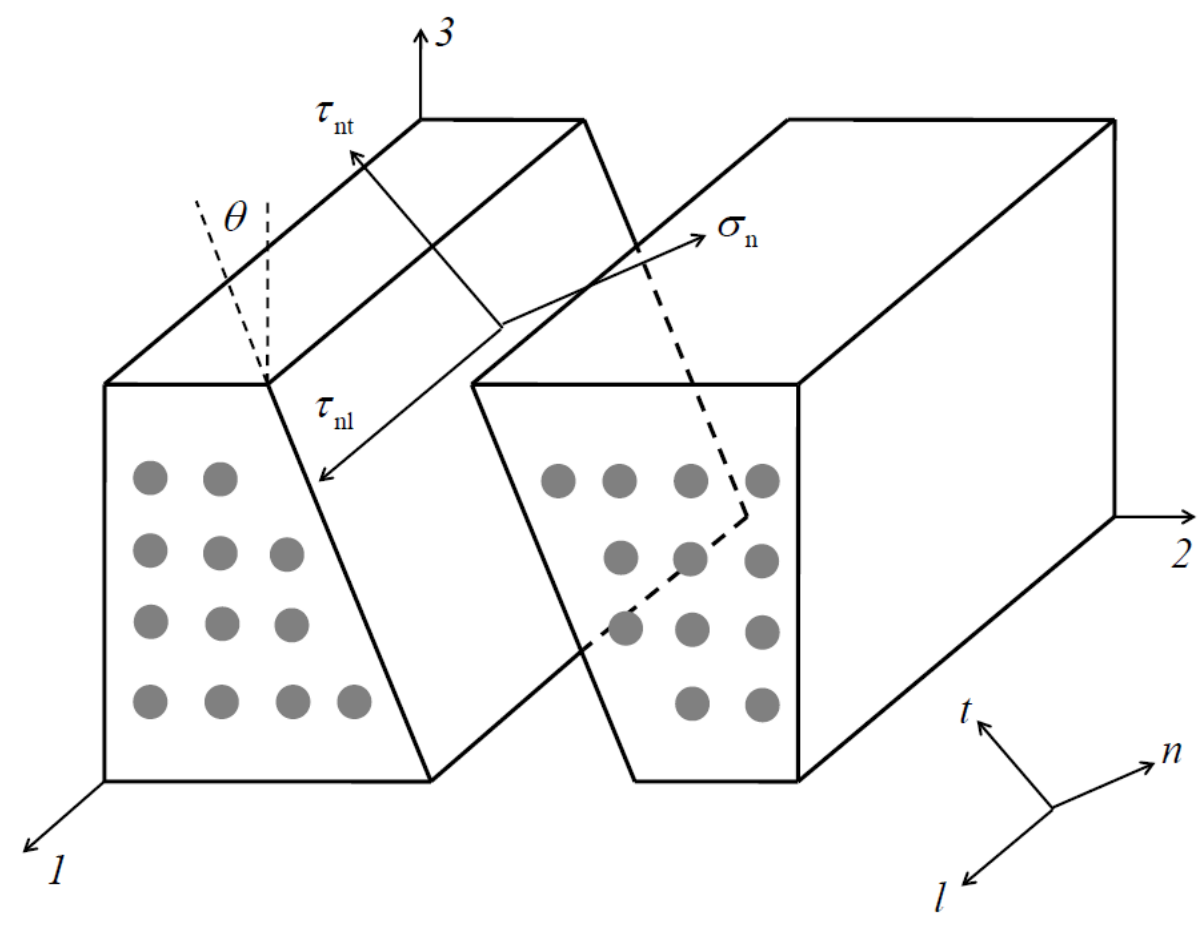

Fig. 1 Definition of stresses acting on the fracture plane.

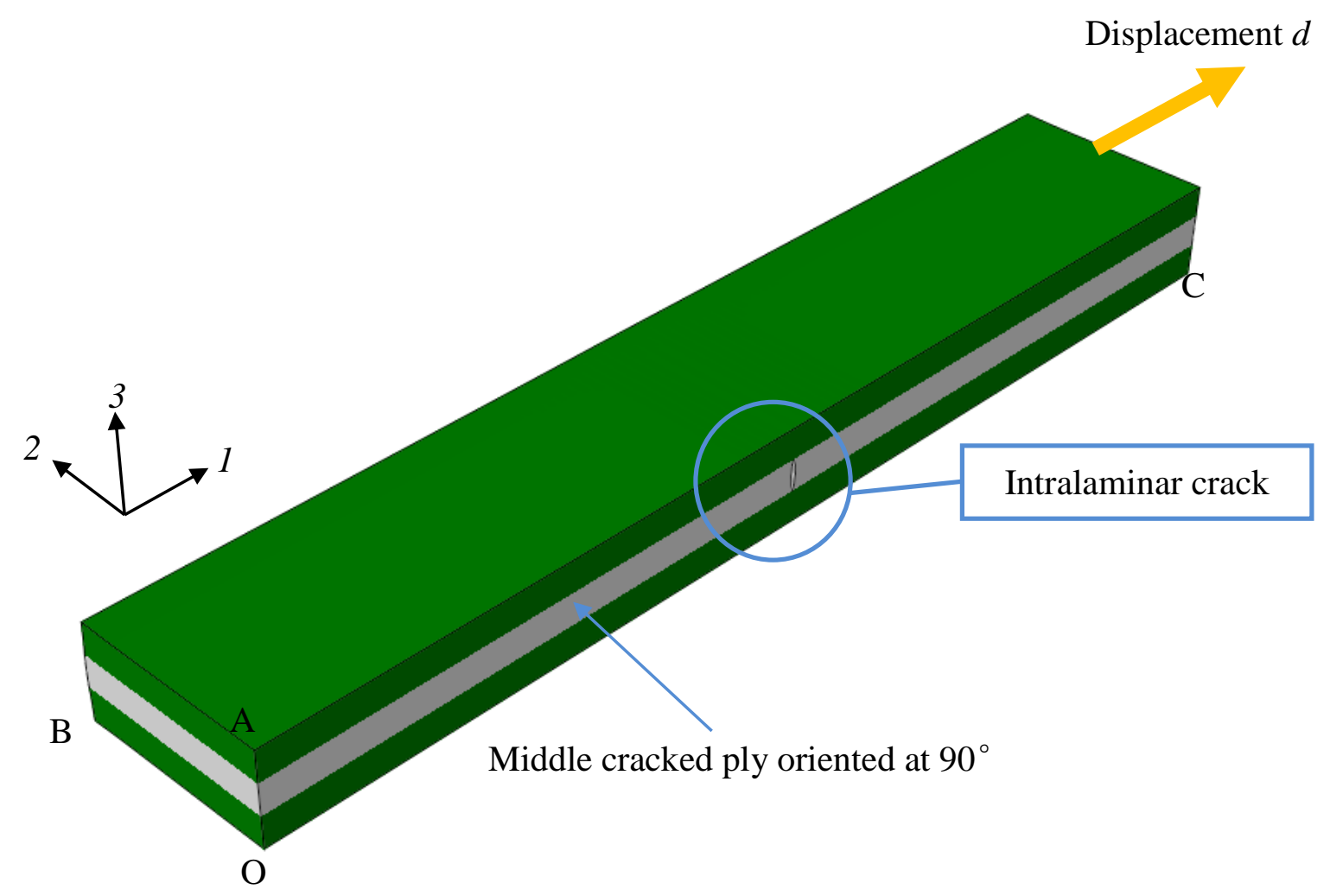

Fig. 2 Schematic of FEM RVE model for computing the saturation crack density. 

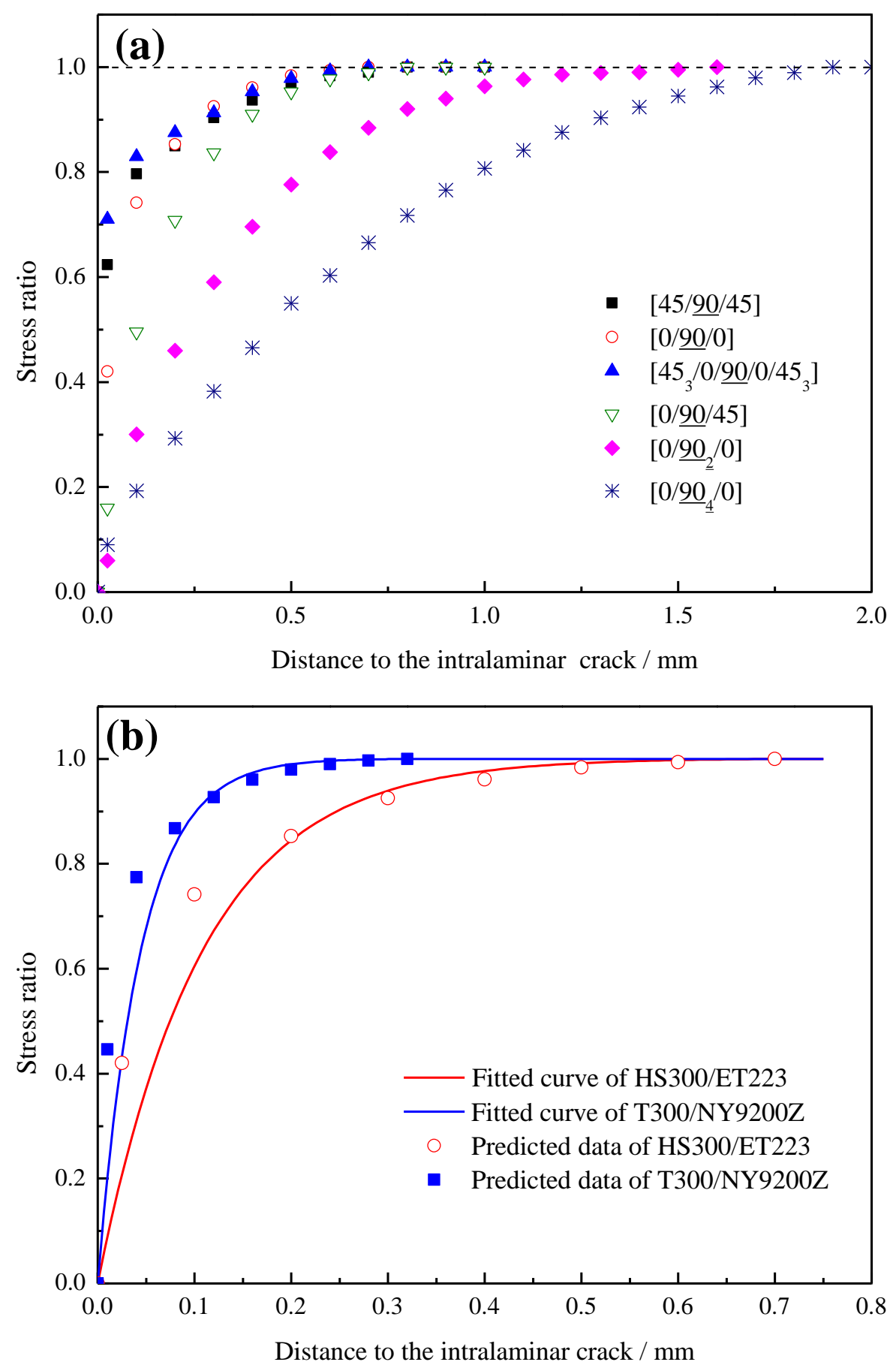

Fig. 3 Distribution of transverse stress in laminates for (a) different stacking sequences and (b) test materials. 


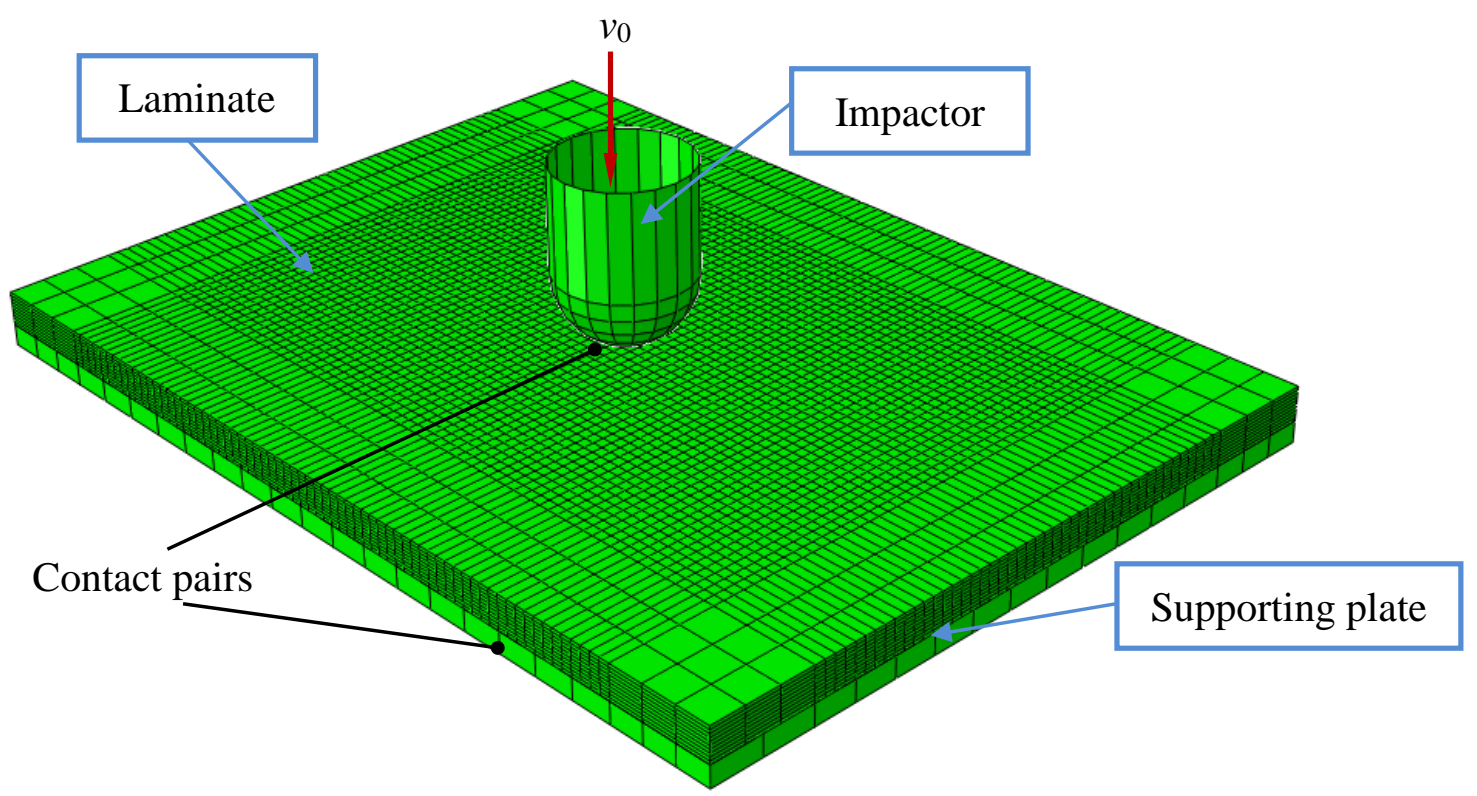

Fig. 4 Low velocity impact FE model of a composite laminate. 

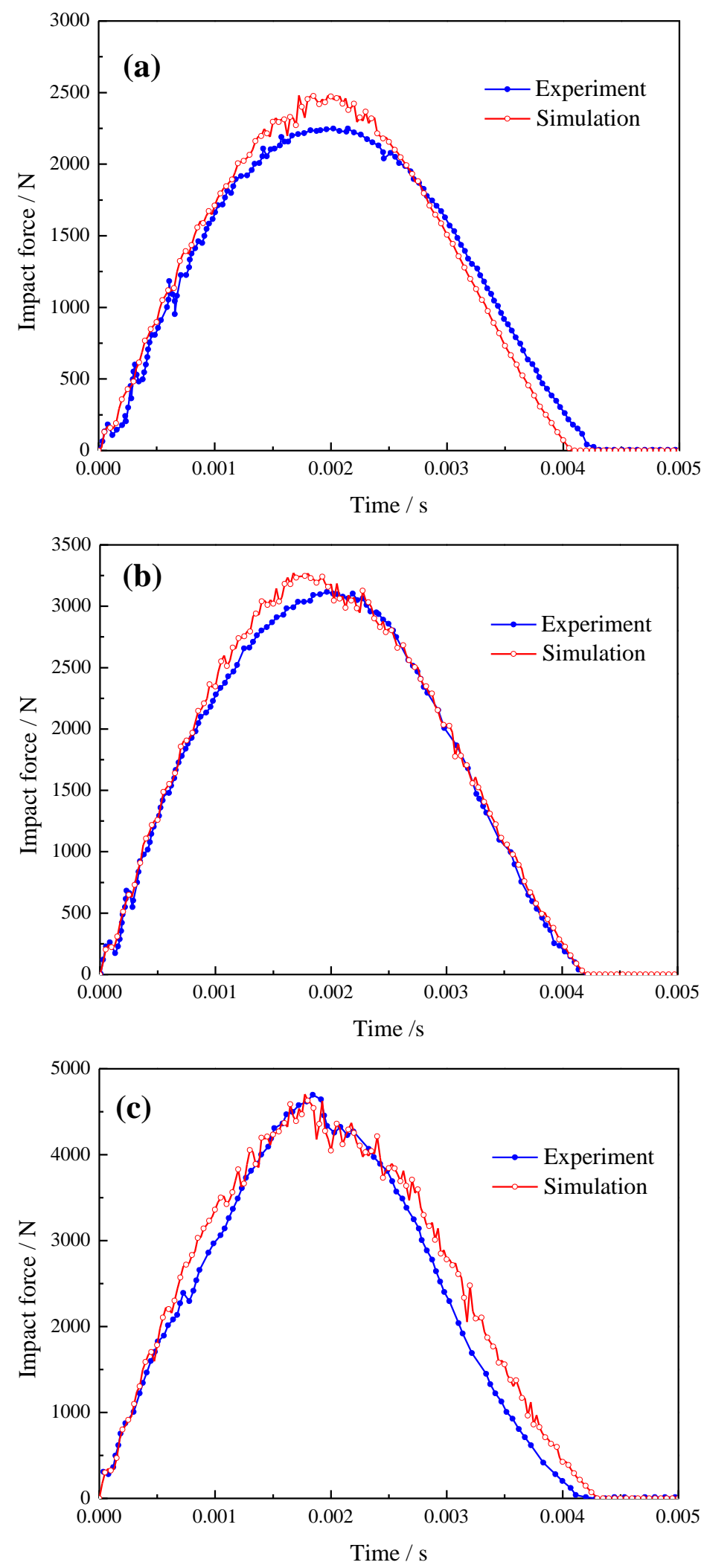

Fig. 5 Comparisons of FEM and measured results of impact force-time histories for laminates $\left[0_{3} / 45 /-45\right]_{\mathrm{S}}$ in test \#1 at the impact energies of (a) $2 \mathrm{~J}$, (b) $4 \mathrm{~J}$ and (c) $8 \mathrm{~J}$. 

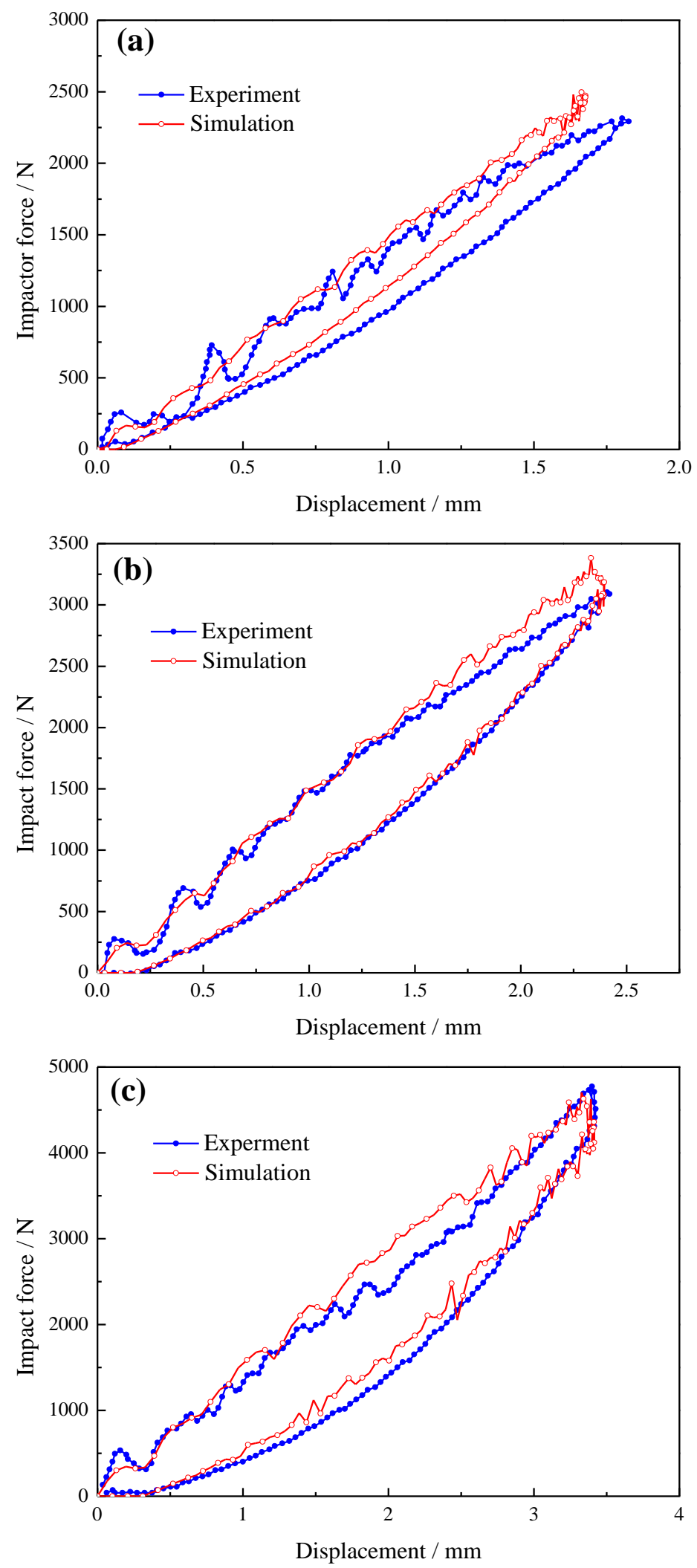

Fig. 6 Comparisons of FEM and measured results of impact force-displacement curves for laminates $\left[0_{3} / 45 /-45\right]_{\mathrm{S}}$ in test \#1 at the impact energies of (a) $2 \mathrm{~J}$, (b) $4 \mathrm{~J}$ and (c) $8 \mathrm{~J}$. 

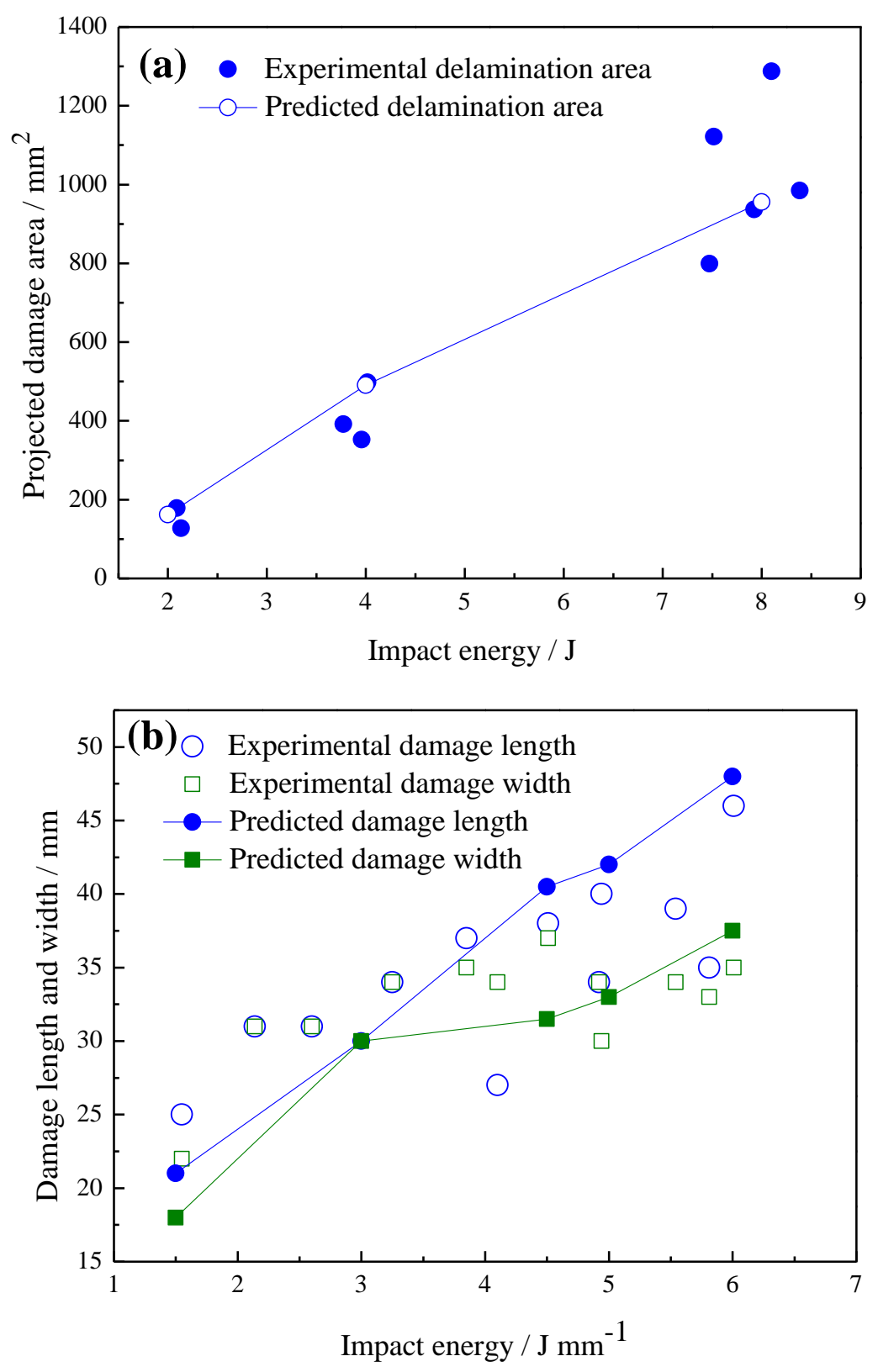

Fig. 7 Comparison between measured and predicted delamination area for (a) laminates $[03 / 45 /-45]_{S}$ in test \#1 and (b) laminates [45/0/-45/90]4S in test \#2 


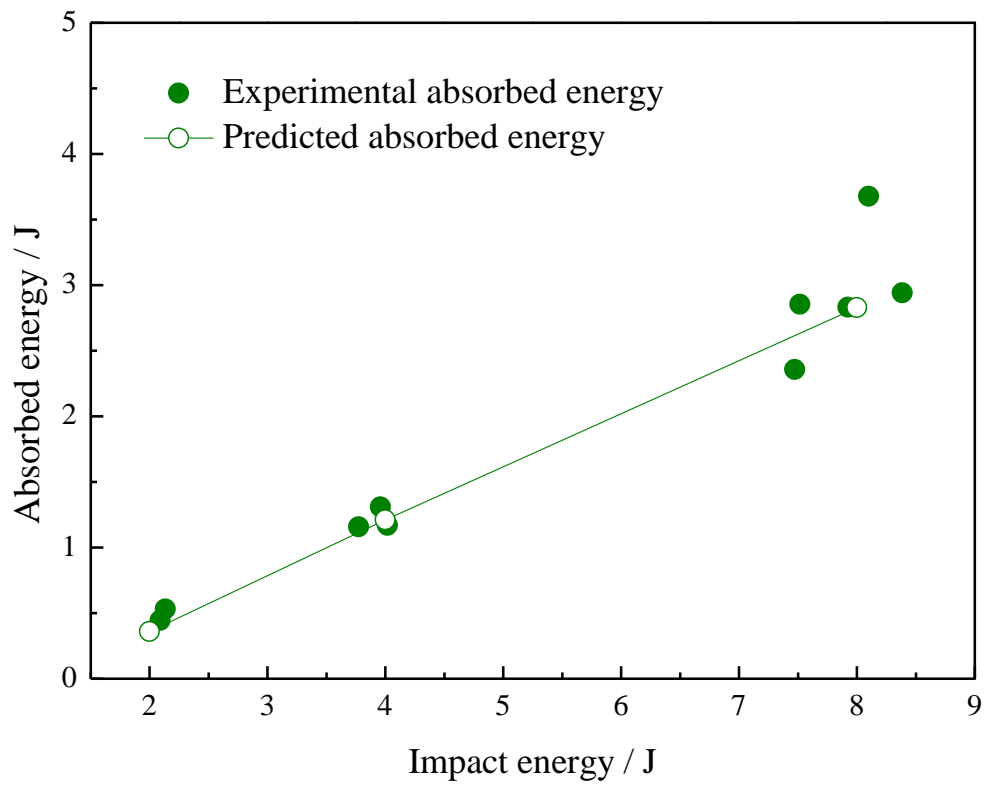

Fig. 8 Comparison between measured and predicted absorbed energy for laminates $\left[0{ }_{3} / 45 /-45\right]_{S}$ in test \#1 


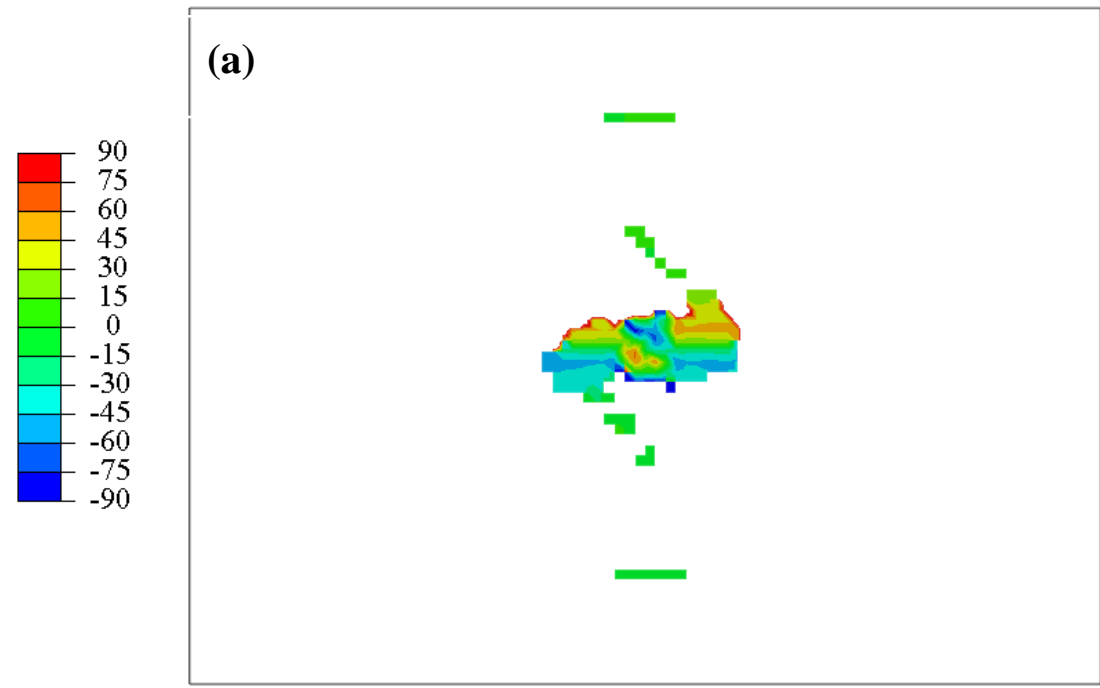

(b)
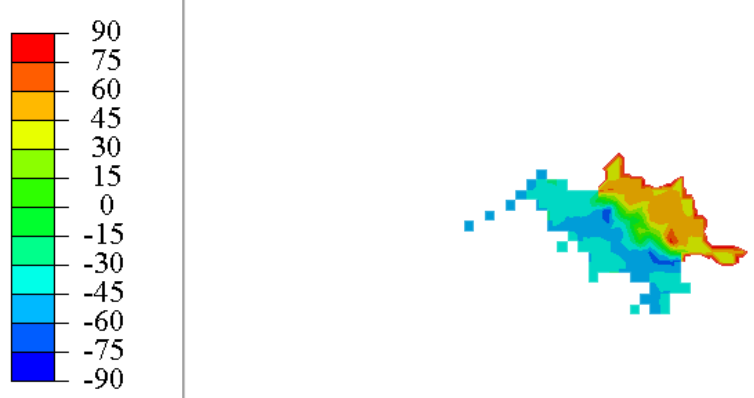

(c)
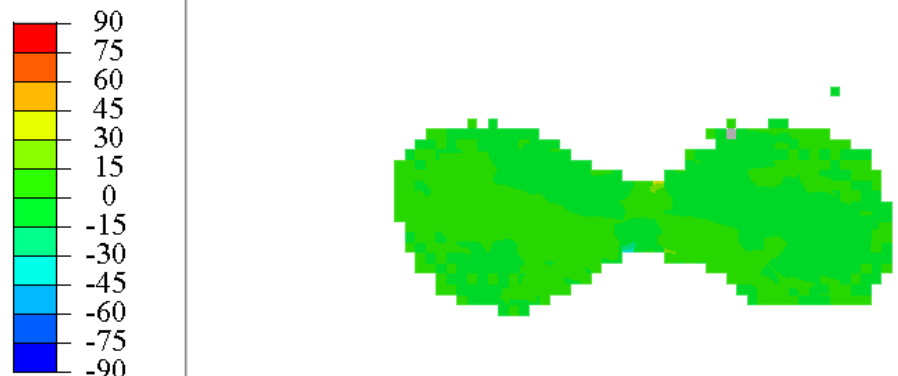

Fig. 9 Distribution of fracture plane angles in typical plies: (a) 1st ply (impact surface),

(b) 5th ply (middle ply) and (c) 10th ply (impact back surface). 

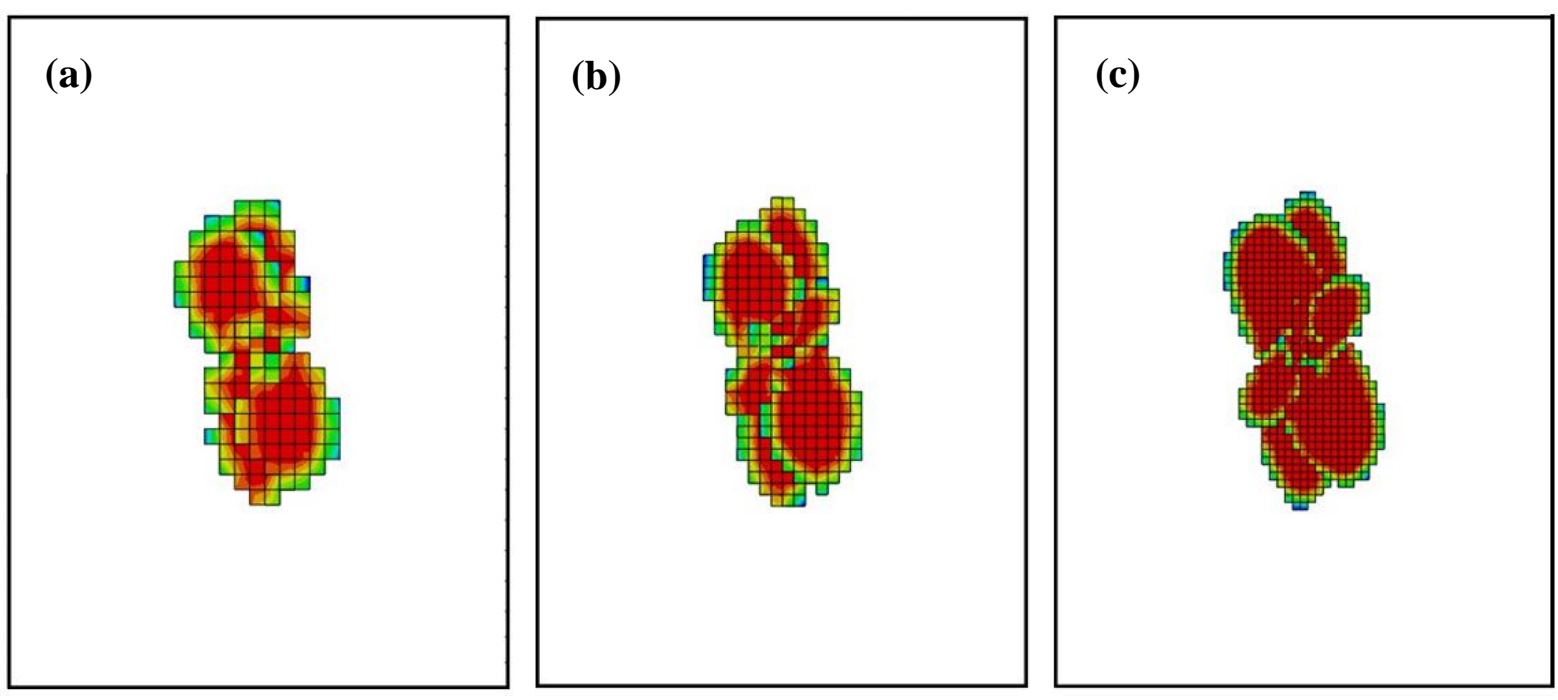

Fig. 10 Numerically projected delamination area for the different mesh densities: (a) 2 $\mathrm{mm} \times 2 \mathrm{~mm}$, (b) $1.5 \mathrm{~mm} \times 1.5 \mathrm{~mm}$ and (c) $1 \mathrm{~mm} \times 1 \mathrm{~mm}$. 

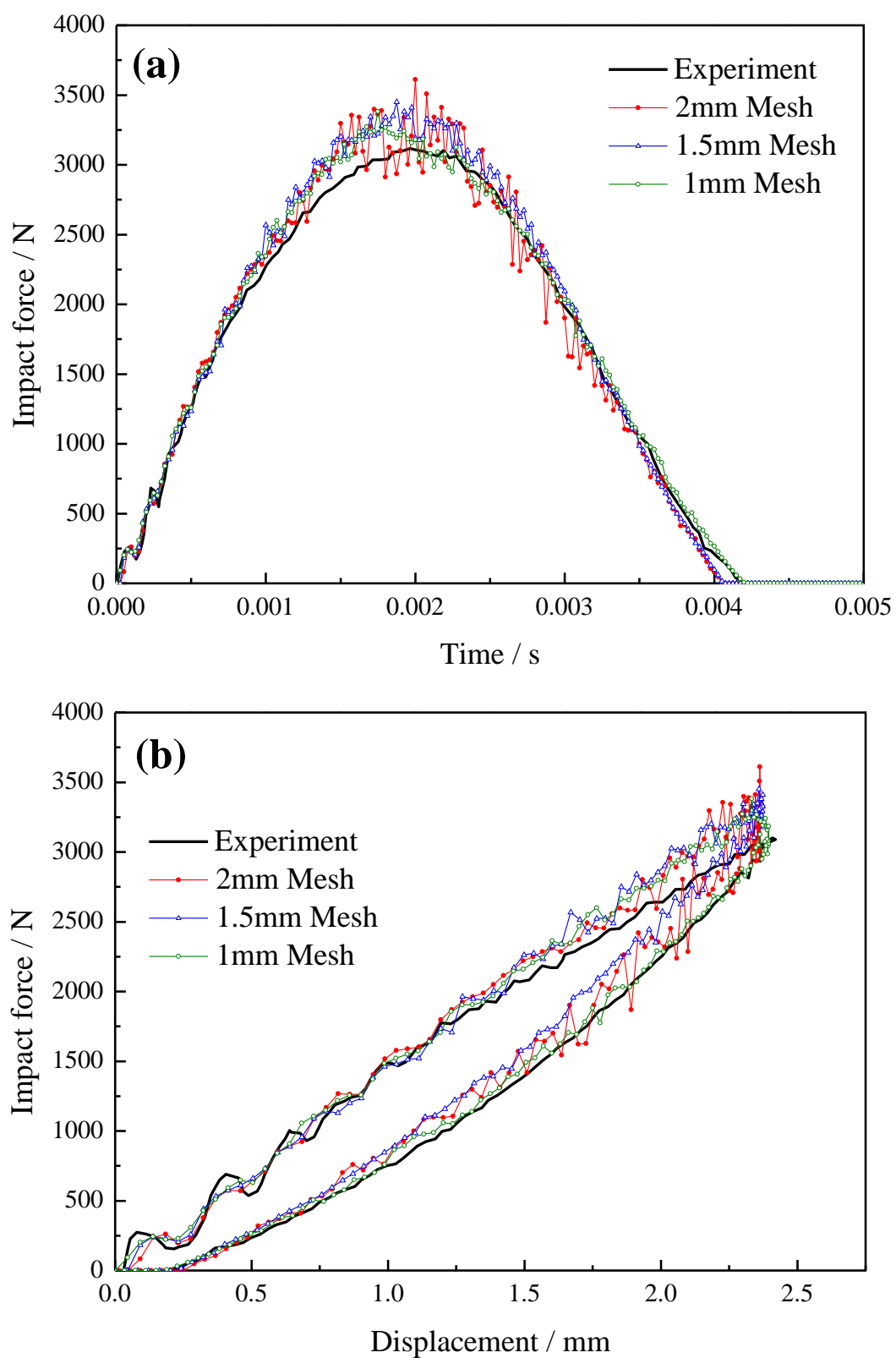

Fig. 11 Predicted (a) impact force-time histories and (b) impact force-displacement curves for the different mesh densities 